\section{Participação da população no controle da dengue: uma análise da sensibilidade dos planos de saúde de municípios do Estado de São Paulo, Brasil}

\author{
Community participation in dengue control: \\ an analysis of the sensitivity of municipal \\ health plans in the State of São Paulo, Brazil
}

\author{
1 Superintendência de \\ Controle de Endemias, \\ São Paulo, Brasil. \\ 2 Departamento de Medicina \\ Social, Faculdade de Ciências \\ Médicas da Santa Casa de \\ São Paulo, São Paulo, Brasil. \\ Correspondência \\ I. T. R. N Ferreira \\ Superintendência de Controle \\ de Endemias. \\ Rua Paula Souza 166, \\ São Paulo, SP \\ 01027000, Brasil. \\ inevez@gmail.com \\ irma@sucen.sp.gov.br
}

\begin{abstract}
Community participation in activities to combat the Aedes aegypti mosquito has been identified as one of the key thrusts in dengue control programs. This study aimed to verify the extent to which local health plans include community participation in dengue control activities in municipalities in the State of São Paulo, Brazil. The municipal health plans were analyzed used a reading script covering their main sections: (i) introduction and analysis of the health situations, (ii) objectives and targets, and (iii) proposed interventions. A sensitivity index was constructed on the basis of affirmative answers. Only one of the 16 health plans showed medium sensitivity to community participation in dengue control, two had a low index, twelve showed a very low index, and one had a zero sensitivity index. The study indicated that community participation fails to occupy a privileged position in municipal health policy for dengue control.
\end{abstract}

Dengue; Communicable Disease Control; Consumer Participation
Irma Teresinha Rodrigues Neves Ferreira 1 Maria Amélia de Sousa Mascena Veras 2 Rubens Antonio Silva ${ }^{1}$

\section{Introdução}

A ocorrência de sucessivas epidemias de dengue em todo o mundo e no Brasil, em particular, vem sendo objeto de inquietação por parte de técnicos, pesquisadores e da sociedade em geral, diante das potenciais conseqüências, como o grau de transmissão generalizada da doença, por todos os meses do ano, com aumento da morbidade e letalidade 1,2 .

No Estado de São Paulo, Brasil, registrou-se, em 2007, a mais elevada incidência da doença desde o início da transmissão no estado, sendo de 219,8/100 mil habitantes. Além do aumento no número de casos e das complicações envolvendo a forma hemorrágica da doença, merece destaque a expansão das áreas geográficas de transmissão que, nos anos de 2006 e 2007, passou de 254 para 369 municípios ${ }^{3}$.

Cerca de $80 \%$ dos municípios paulistas estão infestados pelo inseto transmissor, Aedes aegypti, sendo considerada espécie domiciliada, completamente adaptada ao meio urbano e a diferentes situações ambientais 4 .

Em geral, o combate ao mosquito transmissor da dengue apresenta vários aspectos críticos, como o não cumprimento da legislação ao fiscalizar e eliminar criadouros em pontos considerados estratégicos, por conta de sua importância na dispersão do vetor, a dificuldade de inspeção predial para suprimir ou tratar reservatórios de água, a carência de mão de obra qualificada pa- 
ra vistoria e tratamento nos imóveis, além das limitações legais para contratar pessoal, o que leva à terceirização do trabalho com contratos temporários e precários. Outros aspectos estruturais têm implicação direta no controle do mosquito como a necessidade da regularização do abastecimento público de água, a coleta regular e destinação adequada do lixo, especialmente na periferia dos centros urbanos 5 .

Essas dificuldades remetem à ênfase nas ações de educação e comunicação dirigidas à população a fim de reduzir os criadouros dos mosquitos e a manutenção do ambiente domiciliar saudável. Desse modo, a participação comunitária, de forma consciente e ativa, nas ações de vigilância e controle do Ae. aegypti, tem sido apontada como um dos principais eixos de um efetivo programa de controle, ao mesmo tempo em que se constitui uma das mais complexas tarefas a serem implementadas 6 .

A Organização Pan-Americana da Saúde (OPAS) 7 alerta que um dos maiores obstáculos para efetivar o controle dos focos do mosquito tem sido a falta de habilidade dos órgãos de saúde pública para mobilizar os recursos necessários à consecução e manutenção do impacto sobre o comportamento das populações em situações de risco de dengue e recomenda o modelo de gestão integrado para prevenir e controlar como a estratégia de comunicação para mudança de comportamento dirigida às problemáticas específicas.

O Programa Nacional de Controle de Dengue (PNCD) em vigor no país possui dez componentes que tratam de diferentes estratégias de controle da dengue e um deles apresenta propostas de ações para fomento da participação comunitária como estratégia de controle direcionada à redução de criadouros domiciliares do mosquito 8 .

No entanto, a prevenção e controle das doenças transmissíveis envolvem um conjunto de ações voltadas para os determinantes e condicionantes da saúde, indicando a necessidade de abordagens interdisciplinares e estratégias de políticas públicas integradas na política de saúde de cada localidade.

Dessa forma, o plano de saúde se configura na formalização das intenções e dos resultados a serem buscados pela gestão para que o Sistema Único de Saúde (SUS) responda de forma efetiva, oportuna e com qualidade às demandas e necessidades de saúde da população ${ }^{9}$.

Tendo em vista que a participação popular no controle de dengue é essencial e se constitui em um dos eixos de sustentabilidade de um efetivo programa de vigilância e controle; que o processo participativo não é espontâneo e requer uma intervenção que envolva diferentes atores em sua formulação e execução, dentre eles o poder pú- blico; cabe o pressuposto de que sua implantação deveria estar inserida nos planos municipais de saúde, especialmente em municípios com transmissão de dengue.

Este estudo analisou a importância conferida à inserção da participação popular no controle de dengue pelos formuladores da política municipal de saúde, nos respectivos planos, em municípios selecionados do Estado de São Paulo, no período de 2006 a 2009.

\section{Metodologia}

Recorreu-se, na presente investigação, a uma abordagem descritiva/comparativa que permitiu uma revisão sistemática dos planos municipais de saúde, numa trajetória da apreensão da realidade e do fenômeno de interesse para compreender os significados e a intencionalidade das ações propostas 10 .

Neste sentido, buscando identificar as intenções dos planejadores da saúde quanto à participação da população no controle da dengue, utilizaram-se as etapas básicas da análise de conteúdo, que, por meio de procedimentos sistemáticos e objetivos, permitiu construir indicadores quantitativos que foram utilizados na construção de um índice 11.

Foram utilizados como modelo para formatação e análise os trabalhos de Peiró et al. 12 e Borrell et al. 13, que, estudando as questões de gênero e desigualdades em saúde nos planos de saúde das comunidades autônomas da Espanha, utilizaram um roteiro de leitura e propuseram a construção de um índice de sensibilidade dos planos na abordagem das questões de interesse. Embora sem uma relação explícita com a temática dengue, a abordagem se mostrou adequada aos propósitos deste estudo.

Foram estudados os planos de saúde de municípios do Estado de São Paulo, selecionados de forma intencional, dentre os municípios prioritários para o PNCD, classificados em 2004, pela Secretaria de Estado da Saúde 14, e que são participantes da amostra nacional para Levantamento de Índice Rápido (LIRA).

Na referida classificação, foram priorizados os municípios infestados por Ae. aegypti, com transmissão de dengue, que atendiam a uma das condições descritas a seguir:

1) Ser capital do estado ou sede de região administrativa, ser receptivo à introdução de novos sorotipos de dengue independentemente do porte populacional (portos, aeroportos, núcleos de turismo nacional e / ou internacional);

2) Municípios infestados por Ae. aegypti, com mais de 100 mil habitantes e com transmissão 
de dengue em pelo menos três dos últimos cinco anos; e

3) Municípios infestados por Ae. aegypti, com população entre 100 mil e 50 mil habitantes e com transmissão de dengue em pelo menos três dos últimos cinco anos, com incidência de dengue acumulada no período maior que 300 casos/100 mil habitantes.

Os municípios prioritários aglutinam cerca de $40 \%$ da população paulista e estão localizados nas diferentes regiões administrativas do Estado de São Paulo. Caracterizam-se pela transmissão autóctone da dengue e, em 2007, contribuíram com cerca de $40 \%$ dos casos da doença registrados em todo o estado 5 . Em sua maioria, tais municípios constituem-se em pólos de atração de bens e serviços com intenso deslocamento de pessoas e fluxo migratório.

Dentre eles, optou-se por selecionar aqueles que fossem participantes do LIRA, metodologia de monitoramento dos níveis de infestação do Ae. aegypti, utilizando a avaliação da densidade larvária, proposta pelo Ministério da Saúde. Trata-se de método simplificado de amostragem que fornece índices de maneira rápida e oportuna, permitindo o direcionamento das ações de controle para áreas críticas, além de possibilitar avaliação das atividades desenvolvidas tanto operacionais como de comunicação e mobilização social 15 , razão pela qual esses municípios foram selecionados neste estudo.

Os municípios que atenderam aos dois critérios acima mencionados foram: Araçatuba, Araraquara, Barretos, Bauru, Campinas, Guarulhos, Itu, Marília, Presidente Prudente, Piracicaba, Osasco, Ribeirão Preto, São José do Rio Preto, Santos, São Sebastião e São Paulo.

No período de setembro de 2007 a abril de 2008, buscaram-se as cópias dos documentos municipais, de acordo com as seguintes etapas: pesquisa dos planos disponíveis na Internet; correspondência aos secretários municipais de saúde; contatos com terceiros para intermediação junto aos municípios, além de visitas específicas a municípios com a finalidade de obter cópias dos planos de saúde. Os documentos referiamse ao período de 2006 a 2009 com exceção de Araçatuba, cujo plano referiu-se ao período de 2002-2005, mas vigente atualmente, segundo informações obtidas no município.

Foi elaborado um roteiro de leitura abrangendo as três partes que constituem os planos: (i) introdução e análise da situação de saúde, (ii) objetivos e metas e (ii) propostas de intervenção. A Tabela 1 apresenta o roteiro utilizado na análise dos planos municipais de saúde.

Para cada tema, foram elaboradas três questões, totalizando nove questões, que foram preenchidas com respostas dicotômicas (sim/ não). Foram quantificadas as menções afirmativas para cada questão, dentro de cada tema, de acordo com o número de citações referentes, sendo consideradas três menções como corte, seguindo a classificação proposta pelos autores espanhóis 12,13.

Foram consideradas respostas afirmativas as menções e referências à participação da população no controle da dengue, mediante atividades previstas nos planos, sugestões, propostas e/ ou projetos específicos envolvendo a sociedade civil organizada ou a população em geral, independentemente da forma de participação. Não foram consideradas nas respostas afirmativas as citações que se referiam à participação social nos Conselhos Municipais de Saúde ou nas Conferências Municipais de Saúde, por se constituírem em um tipo de participação que está inserida no quesito controle social e nas exigências legais do SUS, não sendo específica para o controle de doenças.

Com base no número de menções afirmativas, foi construído o índice de sensibilidade que variou de 0 a 5 , sendo considerado $0=$ índice de sensibilidade nulo, quando nenhuma resposta foi afirmativa; 1 = índice muito baixo, quando houve de 1 a 5 menções; 2 = índice baixo, de 6 a 11 menções; 3 = índice médio, para 12 a 16 menções; 4 = índice alto, para 17 a 21 menções e 5 = índice muito alto, quando houve mais de 22 menções. Entendeu-se que quanto maior o número de respostas afirmativas, maior a sensibilidade dos planos à participação popular no controle de dengue. A construção do índice de sensibilidade possibilitou identificar em que medida é contemplada a participação popular nas ações de controle da dengue, em cada município.

Cada plano foi lido duas vezes, pela autora principal e por uma profissional de saúde da área de controle de endemias, observando-se o preenchimento do roteiro. Na verificação dos dados obtidos, as eventuais divergências foram resolvidas de modo consensual.

A revisão dos planos por temas, ou partes, possibilitou a verificação da consistência e da coerência interna do documento, particularmente em relação às intenções expressas, em cada uma das partes, referente ao objeto de estudo. Por outro lado, o agrupamento das informações permitiu obter um panorama das respostas às questões formuladas em cada município, e que se estabelecessem comparações entre elas.

O projeto de investigação foi aprovado pela Comissão Científica do Departamento de Medicina Social da Faculdade de Ciências Médicas da Santa Casa de São Paulo, em 15 de outubro de 2007, que não julgou necessário encaminhá-lo 
Tabela 1

Roteiro de leitura utilizado para coleta de dados.

\begin{tabular}{|c|c|c|c|c|}
\hline \multirow{2}{*}{$\begin{array}{l}\text { Temas } \\
\text { 1. Introdução e análise da situação de saúde }\end{array}$} & \multicolumn{4}{|c|}{ Número/Menções } \\
\hline & 0 & 1 & 2 & 3 \\
\hline \multicolumn{5}{|l|}{$\begin{array}{l}\text { 1.1. Entre os valores e princípios do plano existem referencias à participação da população no controle de doenças e } \\
\text { agravos (dengue)? }\end{array}$} \\
\hline \multicolumn{5}{|l|}{ 1.2. Na descrição e análise da situação municipal de saúde é mencionada a situação da dengue? } \\
\hline \multicolumn{5}{|l|}{$\begin{array}{l}\text { 1.3. Na descrição e análise da situação de saúde é mencionada ou sugerida a participação da população no controle } \\
\text { de doenças e agravos (dengue)? }\end{array}$} \\
\hline \multicolumn{5}{|l|}{ 2. Objetivos e metas } \\
\hline \multicolumn{5}{|l|}{ 2.1. Menciona a participação da população no controle de doenças e agravos (dengue)? } \\
\hline \multicolumn{5}{|l|}{ 2.2. Sugere formas de participação da população no controle de doenças (dengue) e agravos? } \\
\hline \multicolumn{5}{|l|}{ 2.3. Propõe metas dirigidas à inserção da população no controle de doenças (dengue) e agravos? } \\
\hline \multicolumn{5}{|l|}{ 3. Propostas de intervenção } \\
\hline \multicolumn{5}{|l|}{ 3.1. Descreve ações sobre o meio ambiente urbano para o controle de doenças (dengue) e agravos? } \\
\hline 3.2. Propõe linhas de ação para envolvimento e participação da população nos cuidados para o controle da dengue? & & & & \\
\hline 3.3. Inclui mecanismos institucionais que garantam a participação da população no controle do Aedes aegypti? & & & & \\
\hline
\end{tabular}

ao Comitê de Ética em Pesquisa, tendo em vista se tratar de análise documental.

\section{Resultados}

Verificou-se na introdução e análise da situação de saúde que 13 dos 16 planos municipais mencionaram, pelo menos uma vez, a situação da dengue como um dos problemas locais de saúde; sete citaram ou sugeriram a participação popular no controle da dengue e, dentre eles, três fizeram referências aos valores e princípios dos planos (Tabela 2).

Nos objetivos e metas dos planos, verificou-se que dez mencionaram a participação popular no controle de doenças e agravos; cinco referiramse às formas de participação popular no controle de dengue e sete propuseram metas dirigidas à inserção da população no controle de doenças (Tabela 3). Pode-se observar neste item que dentre os municípios que mencionaram a participação popular no controle de doenças, em cinco deles foram sugeridas formas de participação e proposição de metas.

Constatou-se que a maioria dos planos que mencionou participação popular no controle de dengue nos temas anteriores deixou de incorporar atividades na parte relativa às propostas de intervenção (Tabela 4). Nos planos de Campinas, Guarulhos, Osasco, São José do Rio Preto e São Sebastião foram descritas ações sobre o meio ambiente urbano para o controle de doenças.
Nos planos de Santos e São José do Rio Preto, foram mencionadas linhas de ação para o envolvimento e participação popular no controle de dengue e, nos planos de Guarulhos, Osasco e São José do Rio Preto foram incluídos, ao menos uma vez, mecanismos para promover a participação popular no controle de dengue.

Quanto ao índice de sensibilidade, o plano municipal de São José do Rio Preto apresentou índice médio de sensibilidade para a participação da população no controle de dengue, os planos municipais de Osasco e Guarulhos apresentaram índice baixo de sensibilidade, e os demais planos de saúde apresentaram índice muito baixo de sensibilidade, com exceção do plano de Itu, que apresentou índice nulo de sensibilidade. Não houve registro de índice alto e muito alto de sensibilidade nos planos (Tabela 5).

Do ponto de vista da coerência e consistência interna entre as diferentes partes constitutivas dos planos, em relação à participação popular no controle de dengue, identificou-se coerência em quatro planos entre as considerações presentes na introdução e análise da situação de saúde, objetivos e metas, propostas de intervenção. Em seis planos, observaram-se menções à participação popular no controle de dengue na introdução $e$ análise da situação de saúde, objetivos e metas, porém não havia referências nas propostas de intervenção para operacionalizar o objetivo ou meta fixada.

Verificaram-se nos planos de Campinas e Santos que houve referências à participação po- 
Número de menções relativas à participação popular no controle da dengue nos planos de saúde no item introdução e análise da situação de saúde. Estado de São Paulo, Brasil, 2008.

\begin{tabular}{|c|c|c|c|c|}
\hline \multirow[t]{2}{*}{ Municípios } & \multicolumn{4}{|c|}{ Questões referentes à introdução e análise da situação de saúde } \\
\hline & 1.1 & 1.2 & 1.3 & Menções \\
\hline Araçatuba & 0 & 0 & 1 & 1 \\
\hline Araraquara & 0 & 1 & 0 & 1 \\
\hline Barretos & 0 & 1 & 1 & 2 \\
\hline Bauru & 0 & 1 & 0 & 1 \\
\hline Campinas & 0 & 2 & 0 & 2 \\
\hline Guarulhos & 1 & 2 & 0 & 3 \\
\hline Itu & 0 & 0 & 0 & 0 \\
\hline Marília & 0 & 2 & 0 & 2 \\
\hline Piracicaba & 1 & 1 & 0 & 2 \\
\hline Presidente Prudente & 0 & 1 & 0 & 1 \\
\hline Osasco & 0 & 1 & 2 & 3 \\
\hline Ribeirão Preto & 0 & 2 & 0 & 2 \\
\hline Santos & 0 & 1 & 1 & 2 \\
\hline São José do Rio Preto & 2 & 1 & 3 & 6 \\
\hline São Paulo & 0 & 1 & 1 & 2 \\
\hline São Sebastião & 0 & 0 & 1 & 1 \\
\hline
\end{tabular}

Nota: 1.1 Entre os valores e princípios do Plano existem referencias à participação da população no controle de doenças (dengue) e agravos?; 1.2 Na descrição e análise da situação de saúde é mencionada a situação de dengue?; 1.3 Na descrição e análise da situação de saúde é mencionada ou sugerida a participação da população no controle de doenças (dengue) e agravos?

pular na introdução e análise da situação de saúde, propostas de intervenção, sem que houvesse definição de objetivos e metas para as propostas.

Os planos de saúde de Marília, Presidente Prudente e São Paulo assinalaram a situação da dengue no município e a importância da participação popular no controle, mas não estabeleceram objetivos e metas ou propostas de intervenção relacionadas à participação popular no controle de dengue. Em um dos planos não ocorreu nenhuma resposta afirmativa às questões formuladas.

\section{Discussão}

A participação popular no controle de doenças tem sido enfatizada em diretrizes técnicas e propostas de programas de controle que integram estratégias de ação defendidas por organismos nacionais e internacionais ${ }^{8}$. Pode estar inserida na perspectiva de promoção à saúde, que enfatiza a necessidade de capacitação da comunidade para atuar na melhoria da qualidade de vida e da saúde individual e coletiva 16 e que se viabiliza por diferentes estratégias que objetivam o empowerment, mediante propostas de ações que favoreçam o protagonismo da sociedade civil no construir a gestão compartilhada em saúde 17 .

No controle de endemias, a participação popular é apontada como indispensável especialmente onde sua inserção se impõe como condição para o controle, como no caso da dengue, cujo principal vetor associa-se intimamente aos modos de vida e moradia nas áreas urbanas. Por outro lado, a participação comunitária no controle de doenças torna-se limite e potencialidade, ao mesmo tempo, pois as ações de saúde nem sempre se situam de forma ordenada e contínua do ponto de vista operativo, político ou institucional. $\mathrm{O}$ enfrentamento das endemias não apenas admite, mas requer a participação comunitária 7.

O PNCD destaca o apoio ao desenvolvimento de ações educativas com intuito de mudança de comportamento e adoção de práticas para a manutenção do ambiente saudável ${ }^{9}$.

Os resultados obtidos neste estudo, por meio de pesquisa documental, evidenciam que a participação popular nas ações de controle de dengue não ocupa posição privilegiada na formalização da política de saúde municipal. 
Número de menções relativas à participação popular no controle da dengue nos planos de saúde no item objetivos e metas. Estado de São Paulo, Brasil, 2008.

\begin{tabular}{|c|c|c|c|c|}
\hline \multirow[t]{2}{*}{ Municípios } & \multicolumn{4}{|c|}{ Questões referentes aos objetivos e metas } \\
\hline & 2.1 & 2.2 & 2.3 & Menções \\
\hline Araçatuba & 1 & 1 & 1 & 3 \\
\hline Araraquara & 1 & 1 & 1 & 3 \\
\hline Barretos & 1 & 0 & 0 & 1 \\
\hline Bauru & 1 & 0 & 1 & 2 \\
\hline Campinas & 0 & 0 & 0 & 0 \\
\hline Guarulhos & 1 & 0 & 0 & 1 \\
\hline Itu & 0 & 0 & 0 & 0 \\
\hline Marília & 0 & 0 & 0 & 0 \\
\hline Piracicaba & 1 & 0 & 0 & 1 \\
\hline Presidente Prudente & 0 & 0 & 0 & 0 \\
\hline Osasco & 3 & 2 & 1 & 6 \\
\hline Ribeirão Preto & 1 & 0 & 1 & 2 \\
\hline Santos & 0 & 0 & 0 & 0 \\
\hline São José do Rio Preto & 1 & 2 & 2 & 5 \\
\hline São Paulo & 0 & 0 & 0 & 0 \\
\hline São Sebastião & 1 & 1 & 1 & 3 \\
\hline
\end{tabular}

Nota: 2.1 Menciona a participação popular no controle de doenças (dengue) e agravos?; 2.2 Sugere formas de participação popular no controle de doenças (dengue) e agravos?; 2.3 Propõe metas dirigidas à inserção popular no controle de doenças (dengue) e agravos?

Em São José do Rio Preto, classificado como índice médio de sensibilidade, os estudos anteriores que enfocaram a participação popular no controle de dengue e sua associação com o cenário epidemiológico de dengue na localidade, podem ter contribuído para que o município, ao formular suas políticas de intervenção, incorporasse tais resultados no programa municipal de vigilância e controle 18,19.

Já em Osasco e Guarulhos, municípios com uma história recente de transmissão de dengue, cujos planos alcançaram índice baixo de sensibilidade, este resultado pode estar relacionado à concepção ideológica e visão crítica dos formuladores da política de saúde. Como exemplo, no plano de Osasco está proposta a reforma do setor, em alinhamento com os pressupostos básicos do SUS, contemplando a participação de vários atores no debate de ações de promoção à saúde dirigida ao meio ambiente, no estímulo à integração de ações intersetoriais e na abordagem multidisciplinar para qualidade de vida. Constata-se, aqui, uma linha de atuação voltada para uma visão mais ampliada da saúde que requer ações e políticas públicas integradas, e não destaca um problema em particular.
Do mesmo modo, o plano de saúde de Guarulhos faz referência à busca de um sistema eficiente de assistência e promoção da saúde, construído de modo participativo e nesta linha de pensamento aludem a estratégias e projetos prioritários, tais como Circuito da Saúde e Escola Promotora da Saúde, que enfatizam ações de promoção à saúde e mobilização social para melhoria da qualidade de vida dos munícipes, sugerindo avanço na construção compartilhada do conhecimento com base nos saberes científico e popular 20.

Entretanto, ainda que sejam mencionadas nos planos de saúde ações voltadas à participação da população na prevenção de doenças e promoção da saúde, as propostas sugerem ações pontuais, descontínuas e pouco consistentes, com ênfase na divulgação de informações, nos momentos de alta transmissão da doença.

A forma de participação popular é quase sempre no sentido de colaborar com as autoridades sanitárias, e a inspeção é feita pelo agente nos domicílios, o que pode levar à indução de que a responsabilidade pelo controle é do governo. Esta concepção já foi evidenciada em estudo realizado no México, em 2006, sobre participação comunitária na prevenção da dengue, de- 
Número de menções relativas à participação popular no controle da dengue nos planos de saúde no item propostas de intervenção. Estado de São Paulo, Brasil, 2008.

\begin{tabular}{|c|c|c|c|c|}
\hline \multirow[t]{2}{*}{ Municípios } & \multicolumn{4}{|c|}{ Questões referentes às Propostas de intervenção } \\
\hline & 3.1 & 3.2 & 3.3. & Menções \\
\hline Araçatuba & 0 & 0 & 0 & 0 \\
\hline Araraquara & 0 & 0 & 0 & 0 \\
\hline Barretos & 0 & 0 & 0 & 0 \\
\hline Bauru & 0 & 0 & 0 & 0 \\
\hline Campinas & 3 & 0 & 0 & 3 \\
\hline Guarulhos & 1 & 0 & 3 & 4 \\
\hline Itu & 0 & 0 & 0 & 0 \\
\hline Marília & 0 & 0 & 0 & 0 \\
\hline Piracicaba & 0 & 0 & 0 & 0 \\
\hline Presidente Prudente & 0 & 0 & 0 & 0 \\
\hline Osasco & 2 & 0 & 1 & 3 \\
\hline Ribeirão Preto & 0 & 0 & 0 & 0 \\
\hline Santos & 0 & 1 & 0 & 1 \\
\hline São José do Rio Preto & 3 & 1 & 1 & 5 \\
\hline São Paulo & 0 & 0 & 0 & 0 \\
\hline São Sebastião & 3 & 0 & 0 & 3 \\
\hline
\end{tabular}

Nota: 3.1 Descreve ações sobre o meio ambiente urbano para controle de doenças (dengue) e agravos?; 3.2 Propõe linhas de ação para envolvimento e participação popular no controle da dengue?; 3.3 Inclui mecanismos institucionais que garantam a participação popular no controle da dengue?

monstrando que a população responsabiliza o governo e o governo responsabiliza a população pelo fracasso do programa de controle 21 .

Durante as epidemias, observam-se fortes pressões do poder público e da mídia causando grande comoção popular, mas, à medida que diminui a intensidade da transmissão, a dengue cai no esquecimento, esvazia-se toda a participação, a ameaça e os riscos ficam para o próximo verão 22 .

Mais da metade dos planos analisados apresentou um índice muito baixo de sensibilidade para a participação popular nas ações de controle de dengue, o que poderia constituir-se um paradoxo, considerando que parte desses municípios tem uma contribuição importante na história epidemiológica da dengue no Estado de São Paulo, sobretudo Campinas e Santos, que registram transmissão de dengue há mais de dez anos. No Município de Ribeirão Preto, por exemplo, foi onde ocorreu no final de 1990, a primeira epidemia de grande dimensão no estado, que se espalhou, na época, para outros 62 municípios. Desde então o município experimentou várias epidemias de dengue e em seu plano de saúde não são mencionadas ações voltadas à promoção da saúde e participação da sociedade no controle de dengue.

Uma das propostas do projeto Cidades Saudáveis, destacada na Agenda 21, é promover o desenvolvimento institucional e o fortalecimento da capacidade de planejamento da cidade, incorporando no processo a dimensão ambiental e assegurando a efetiva participação da sociedade, o que requer um compromisso político das autoridades constituídas 23 . As ações para o controle das doenças como a dengue não podem estar dissociadas das questões relativas ao meio ambiente, das questões estruturais e nem restritas ao setor saúde, devendo ser incorporadas no planejamento local com previsão de recursos para sua operacionalização.

Embora esta análise, feita com base em documentos, ratifique que a dengue é um problema de saúde para os municípios incluídos no estudo e que há necessidade de adoção de medidas de controle para seu enfrentamento, não foram encontradas correspondências entre este posicionamento e a definição dos objetivos, metas e propostas de intervenção relacionadas à participação popular. 
Índice geral de sensibilidade dos planos de saúde de municípios do Estado de São Paulo, Brasil, 2008.

\begin{tabular}{|c|c|c|c|c|c|}
\hline \multirow[t]{2}{*}{ Municípios } & \multicolumn{5}{|c|}{ Estrutura básica dos planos de saúde } \\
\hline & $\begin{array}{l}\text { Introdução } \\
\text { e análise da } \\
\text { situação de } \\
\text { saúde }\end{array}$ & $\begin{array}{l}\text { Objetivos e } \\
\text { metas }\end{array}$ & $\begin{array}{l}\text { Propostas de } \\
\text { intervenção }\end{array}$ & Total * & $\begin{array}{c}\text { Índice de } \\
\text { sensibilidade }\end{array}$ \\
\hline Araçatuba & $1 / 9$ & $3 / 9$ & $0 / 9$ & $4 / 27$ & 1 \\
\hline Araraquara & $1 / 9$ & $3 / 9$ & $0 / 9$ & $4 / 27$ & 1 \\
\hline Barretos & $2 / 9$ & $1 / 9$ & $0 / 9$ & $3 / 27$ & 1 \\
\hline Bauru & $1 / 9$ & $2 / 9$ & $0 / 9$ & $3 / 27$ & 1 \\
\hline Campinas & $2 / 9$ & $0 / 9$ & $3 / 9$ & $5 / 27$ & 1 \\
\hline Guarulhos & $3 / 9$ & $1 / 9$ & $4 / 9$ & $8 / 27$ & 2 \\
\hline Itu & $0 / 9$ & $0 / 9$ & $0 / 9$ & $0 / 27$ & 0 \\
\hline Marília & $2 / 9$ & $0 / 9$ & $0 / 9$ & $2 / 27$ & 1 \\
\hline Piracicaba & $2 / 9$ & $1 / 9$ & $0 / 9$ & $3 / 27$ & 1 \\
\hline Presidente Prudente & $1 / 9$ & $0 / 9$ & $0 / 9$ & $1 / 27$ & 1 \\
\hline Osasco & $3 / 9$ & $6 / 9$ & $3 / 9$ & $12 / 27$ & 2 \\
\hline Ribeirão Preto & $2 / 9$ & $2 / 9$ & $0 / 9$ & $4 / 27$ & 1 \\
\hline Santos & $2 / 9$ & $0 / 9$ & $1 / 9$ & $3 / 27$ & 1 \\
\hline São José do Rio Preto & $6 / 9$ & $5 / 9$ & $5 / 9$ & $16 / 27$ & 3 \\
\hline São Paulo & $2 / 9$ & $0 / 9$ & $0 / 9$ & $2 / 27$ & 1 \\
\hline São Sebastião & $1 / 9$ & $3 / 9$ & $3 / 9$ & $7 / 27$ & 1 \\
\hline
\end{tabular}

* Número de resposta afirmativa/Número de respostas possíveis.

Tais considerações, além de refletir o grau de importância conferido à participação popular como estratégia de controle de dengue, podem indicar um provável comprometimento da qualidade técnica, quando da elaboração dos planos municipais de saúde, refletindo dificuldades na formulação do plano ou de conciliação entre as prioridades, as políticas e as lideranças, ou mesmo desarticulação entre as diferentes áreas técnicas e a coordenação.

Cabe observar que o índice de sensibilidade, baseado nos planos municipais de saúde, pode não refletir a situação real de cada município, já que o plano tem um caráter mais genérico e abrangente, e alguns municípios explicitam em documentos complementares os programas específicos para controle de doenças, incluindo a dengue. Por outro lado, uma das motivações para verificar a sensibilidade dos planos municipais quanto à participação popular no controle de dengue foi a suposição de que, se uma proposta de ação constar nos planos de saúde, é mais provável sua viabilização, por meio da mobilização de recursos humanos e financeiros.

Ainda que as ações de participação comunitária sejam priorizadas, avalia-se como pertinente pensar nas possibilidades e desafios operacionais da atual organização do setor saúde mais centrados na doença do que na saúde, resultando na dicotomia entre as ações curativas e preventivas. Em alguns planos, é possível perceber a existência de uma relação vertical com a clientela, a participação comunitária é destacada como necessária de modo colaborativo, não se distinguindo o potencial que a responsabilidade compartilhada entre poder público e sociedade pode representar. Alem disso, não se constata a valorização do saber popular ou espaço para sua incorporação, parecendo subestimar a potencialidade de trabalhar junto e com base nele, especialmente quando a efetividade de um programa depende substancialmente da adesão popular 20,24.

Por outro lado, possivelmente até como resposta, a população se posiciona de forma passiva, receptiva e dependente, num reflexo da própria cultura e do processo histórico de formação 6 .

A falta de planejamento de ações de intervenção que contemplem a participação comunitária como um processo educacional, visando à autonomia dos sujeitos numa perspectiva histórica, pedagógica e politicamente adequada, implica a falta de capacitação dos profissionais de saúde municipais para lidar com os fatores sociais que favorecem a transmissão de dengue. 
Assim, interroga-se sobre o grau de sensibilização e motivação das pessoas para a ação, indaga-se sobre as competências técnicas requeridas dos profissionais de saúde para ter clareza do sentido de sua prática, e qual a possibilidade de re-significar o processo pedagógico que permeia as ações preventivas no controle de doenças.

Por outro lado, a inserção de atores sociais oriundos de diferentes segmentos possibilita novos olhares e contribuições, e pode conduzir a outra lógica de atuação centrada nas pessoas, no estabelecimento de vínculos entre profissionais e população e na construção coletiva do conhecimento. A utilização do espaço social nas unidades de saúde, por exemplo, pode incentivar a identificação, o debate e a compreensão dos problemas ou necessidades de saúde, considerando que ensinar é, sobretudo, criar possibilidades para produção do conhecimento, pois o homem não é completo; por isso está sempre aberto a mudanças e apto a incorporar novos saberes 25 .

Nesta perspectiva, uma mudança de olhar na gestão setorial resultaria em programas de ações que objetivassem o desenvolvimento da consciência sanitária dos grupos populacionais pela compreensão dos problemas de saúde e de seus determinantes, além de influir positivamente na instituição de responsabilidade social compartilhada e no exercício da cidadania 26.

O planejamento de programas de educação em saúde e de participação comunitária para controle de dengue requer direção, financiamento e trabalho especializado que inclua abordagem multiprofissional e integração de ações intersetoriais coordenadas, com envolvimento dos segmentos sociais e de profissionais da área de comunicação, alicerçando grandes parcerias na utilização da mídia e fomentando a participação comunitária 27 .

Estudos realizados, em diferentes localidades, demonstraram que a população tem níveis satisfatórios de conhecimento sobre dengue, formas de transmissão e medidas de controle como resultado das campanhas e outras atividades informativas, mas isso não se reflete na conduta de manutenção do ambiente doméstico livre de criadouros do Ae. aegypti 18,28.

Esse descompasso entre o conhecimento e a prática ou a falta de correspondência entre o saber instituído e a mudança de comportamento pode ser atribuído ao fato de que o comportamento das pessoas não é fruto apenas da assimilação de seus conhecimentos, mas também de suas percepções, valores, representações, crenças e sentimentos, que não podem ser modificados unicamente por meio de acesso ao conhecimento. O entendimento dessas significações é fundamental para balizar a ação educativa que se pretende realizar. Decorre daí a necessidade de repensar a prática pedagógica e a estratégia de ação que informa, mas não forma, cidadãos ativos e conscientes 29,30 e incluir na política de saúde local estratégias e mecanismos que garantam o desencadeamento de ações dirigidas aos profissionais e à população em uma linha transformadora e inclusiva.

A reflexão sobre os índices de sensibilidade obtidos nos planos municipais de saúde, em relação ao controle de dengue, estimulou a busca de outros referenciais teóricos que articulassem aspectos similares à metodologia desenvolvida pelos autores espanhóis. O modelo de vulnerabilidade, proposto por Mann \& Tarantola 31, que consideram a vulnerabilidade à infecção pelo HIV/AIDS foi considerado adequado. O conceito de vulnerabilidade diz respeito à chance de exposição das pessoas ao adoecimento como resultante de um conjunto de fatores individuais, sociais e programáticos. Os fatores programáticos englobam o acesso e a forma de organização dos serviços de saúde, mas também a existência de programas voltados à prevenção, à assistência e ao controle. O conceito de vulnerabilidade programática foi construído referindo-se aos programas de controle de doenças, envolvendo grau de compromisso das instituições, recursos, gerência e monitoramento dos programas nos diferentes níveis de atenção 32 .

Assim, ressalvadas as diferenças de abordagem, o índice de sensibilidade tem correspondência com a vulnerabilidade programática, reflete em que medida os planos municipais priorizam a participação comunitária, enquanto estratégia de controle e em que medida tal priorização remete à organização do sistema para reduzir situações de risco em cada localidade, promover a saúde e envolver a população nas ações de controle de dengue.

Estudioso do campo ${ }^{33}$, refletindo sobre a epidemia de dengue, identifica a necessidade de repensar o modelo de comunicação social atualmente em vigor, voltado particularmente à participação individual, considerando que a dengue, antes de ser uma doença do indivíduo, é principalmente um problema coletivo. Enfatiza que divulgar de forma sistemática e oportuna informações que permitam identificar as áreas de risco, níveis de infestação e recipientes predominantes para proliferação do mosquito podem contribuir para manter a mobilização da população e do setor público.

Deve-se entender que essas ações, de forma isolada, podem não reduzir o risco de novas epidemias porque há outros aspectos relevantes para o controle da doença, mas, localmente, 
compõem um conjunto de medidas que podem e devem ser planejadas de forma integrada e sustentável.

\section{Considerações finais}

A construção dos índices de sensibilidade com base em planos de saúde de municípios no Estado de São Paulo pode ser útil para discutir o controle de dengue, indagando em que medida a baixa ou alta sensibilidade contribui para maior ou menor vulnerabilidade da população a situações de risco de adoecer e morrer, especialmente na perspectiva de aumento das formas graves da doença.

Os planos municipais de saúde são um instrumento de gestão da política municipal de saúde e representam a resposta do setor às ne- cessidades da população, mesmo considerando que há dissonância entre as propostas formuladas e sua efetivação, o que vem a constituir uma limitação dos estudos restritos à análise de documentos oficiais.

Ainda assim, importa avaliar criteriosamente os programas e ações vigentes em âmbito local e corrigir ou propor metas que contribuam para redução das condições de vulnerabilidade programática bem como enfatizem a responsabilidade compartilhada entre poder público e sociedade.

A complexa tarefa de evitar epidemias de dengue implica um conjunto de medidas de natureza política, técnica e social, que pela sua magnitude potencial de transmissão e transcendência extrapola os limites do setor saúde. A opção de investir na participação comunitária poderá se traduzir em ação estruturante da vigilância em saúde e em estratégia de controle mais efetiva.

\section{Resumo}

A participação da população nas ações de combate ao Aedes aegypti tem sido apontada como um dos principais eixos dos programas de controle de dengue. Este estudo objetivou verificar em que medida os planos municipais de saúde contemplam a participação da população nas ações de controle de dengue, em municípios do Estado de São Paulo, Brasil. A análise dos planos de saúde foi realizada com base em um roteiro de leitura abrangendo suas partes constitutivas: (i) a introdução e análise da situação de saúde, (ii) objetivos e metas e (iii) propostas de intervenção. Com base nas menções, foi construído um índice de sensi- bilidade. Apenas um dos 16 planos de saúde analisados apresentou o índice médio de sensibilidade para a participação da população no controle de dengue, dois apresentaram índice baixo, 12 planos apresentaram índice muito baixo e um apresentou índice nulo de sensibilidade. Tal estudo permitiu identificar que a participação da população não ocupa posição privilegiada na formulação da política municipal de saúde para o controle de dengue.

Dengue; Controle de Doenças Transmissíveis; Participação Comunitária 


\section{Colaboradores}

I. T. R. N. Ferreira participou de todas as etapas, desde a concepção e planejamento da pesquisa, coleta e análise dos dados até a redação final do artigo. M. A. S. M. Veras colaborou em todas as etapas do estudo, desde o seu planejamento até a análise dos dados e redação final do artigo. R. A. Silva contribuiu nas etapas do estudo, da análise e interpretação dos dados e redação do artigo.

\section{Agradecimentos}

À Superintendência de Controle de Endemias que permitiu cumprir os créditos do curso e suas decorrências. Aos municípios selecionados que cederam cópias dos respectivos planos de saúde. À Dalva Marli Valério Wanderley pelas significativas contribuições em todo processo de trabalho. À Flora Barbosa Telles pela valiosa colaboração nas leituras, sugestões, amizade e companheirismo.

\section{Referências}

1. Teixeira MG, Costa MCN, Barreto, ML. Epidemiologia e medidas de prevenção do dengue. Inf Epidemiol SUS 1999; 8:5-33.

2. Gubler DJ, Clark GG. Community based integrate control of Aedes aegypti: a brief overview of currents programs. Am J Trop Med Hyg 1994; 50: 50-60.

3. Centro de Vigilância Epidemiológica "Prof. Alexandre Vranjac". Boletim de Dengue: casos autóctones confirmados por município e semana epidemiológica - anos 2006, 2007 e 2008. http://www.cve. saude.sp.gov.br/htm/cve_dengue.html (acessado em 19/Jun/2008).

4. Marques GM, Arduino MB, Serpa LLN, Gomes AHA, Pereira M. Programa de controle do Aedes aegypti no Estado de São Paulo. Boletim Epidemiológico Paulista 2006; 3 Suppl 1:36-9.

5. Tauil PL. Aspectos críticos do controle da dengue no Brasil. Cad Saúde Pública 2002; 18:867-71.

6. Dias JCP. Problemas e possibilidades de participação comunitária no controle das grandes endemias no Brasil. Cad Saúde Pública. 1998; 14 Suppl 2: 19-37.
7. Organização Pan-Americana da Saúde. Diretrizes relativas à prevenção e ao controle do dengue e do dengue hemorrágico nas Américas. Washington DC: Organização Pan-Americana da Saúde; 1991.

8. Fundação Nacional de Saúde. Programa Nacional de Controle da Dengue (PNCD). Brasília: Fundação Nacional de Saúde; 2002.

9. Coordenação Geral de Planejamento, Subsecretaria de Planejamento e Orçamento, Secretaria Executiva, Ministério da Saúde. Organização e funcionamento do sistema de planejamento do SUS. Brasília: Ministério da Saúde; 2006. (Série B Textos Básicos de Saúde).

10. Minayo MCS. O desafio do conhecimento: pesquisa qualitativa em saúde. São Paulo: Editora Hucitec; 1992.

11. Bardin L. Análise de conteúdo. Lisboa: Edições 70; 1994.

12. Peiró R, Nieves R, Alvarez-Dardet C, Colomer C, Moya C, Borrell C, et al. Sensibilidad de género en la formulación de planes de salud en España: lo que pudo ser y no fue. Gac Sanit 2004; 18 Suppl 2:36-46. 
13. BorrellC, Peiró R, Ramón N, Pasarína MI, ColomerC, Zafra E, et al. Desigualdades socioeconómicas y planes de salud en las comunidades autónomas del Estado español. Gac Sanit 2005; 19:277-85.

14. Secretaria de Estado da Saúde. Programa de Controle de Dengue no Estado de São Paulo: seleção de municípios prioritários - 2005. Documento técnico. São Paulo: Superintendência de Controle de Endemias/Divisão de Zoonose, Centro de Vigilância Epidemiológica "Prof. Alexandre Vranjac"; 2005.

15. Diretoria Técnica de Gestão, Secretaria da Vigilância em Saúde, Ministério da Saúde. Diagnóstico rápido nos municípios para vigilância entomológica de Aedes aegypti no Brasil - LIRAa: metodologia para avaliação dos índices de Breteau e Predial. Brasília: Ministério da Saúde; 2005. (Série A - Normas e Manuais Técnicos).

16. Organização Mundial da Saúde. Carta de Ottawa. http://www.opas.org.br/promocao/uploadArq/ Ottawa.pdf (acessado em 20/Jul/2008).

17. Stotz EN, Araújo JWG. Promoção da saúde e cultura política: a reconstrução do consenso. Saúde Soc 2004; 13:5-19.

18. Chiaravalloti Neto F, Moraes MS, Fernandes MA, Avaliação dos resultados de atividades de incentivo à participação da comunidade no controle da dengue em um bairro periférico do Município de São José do Rio Preto, São Paulo, e da relação entre conhecimentos e práticas desta população. Cad Saúde Publica 1998; 14 Suppl 2:101-9.

19. Chiaravalloti Neto F, Baglini V, Cesarino MB, Favaro EA, Mondini A, Ferreira AC, et al. O Programa de Controle da Dengue em São José do Rio Preto, São Paulo, Brasil: dificuldades para a atuação dos agentes e adesão da população. Cad Saúde Pública 2007; 23:1656-64

20. Oliveira RM, Valla VV. As condições e as experiências de vida de grupos populares no Rio de Janeiro: repensando a mobilização popular no controle da dengue. Cad Saúde Pública 2001; 17 Suppl:77-88.

21. Toledo-Romaní ME, Baly-Gil A, Ceballos-Ursula E, Boelaert M, van der Stuyft P. Participación comunitaria en la prevención del dengue: un abordaje desde la perspectiva de los diferentes actores sociales. Salud Pública Méx 2006; 48:39-44.
22. Sabroza PC, Toledo LM, Osanai CH. A organização do espaço e os processos endêmicos epidêmicos. In: Leal MC, Sabroza PC, Rodrigues RH, Buss PM, organizadores. Saúde, ambiente e desenvolvimento. v. 2. São Paulo: Editora Hucitec/Rio de Janeiro: ABRASCO; 1992. p. 57-77.

23. Ferraz ST. Cidades saudáveis. uma urbanidade para 2000. Brasília: Paralelo 15; 1999.

24. Mohr A, Schall VT. Rumos da educação em saúde no Brasil e sua relação com a educação ambiental. Cad Saúde Pública 1992; 8:199-203.

25. Freire P. Pedagogia da autonomia: saberes necessários à prática educativa. São Paulo: Editora Paz e Terra; 1996.

26. Buss PM. Promoção da saúde e qualidade de vida. Ciênc Saúde Coletiva 2000; 5:163-77.

27. Martinez Torres E. Dengue. Rio de Janeiro: Editora Fiocruz; 2005

28. Donalisio MR, Alves MJ, Visockas. Inquérito sobre conhecimentos e atitudes da população sobre transmissão do dengue: região de Campinas, São Paulo, Brasil, 1998. Rev Soc Bras Med Trop 2001; 34:197-201.

29. Alves VS. Um modelo de educação em saúde para o Programa Saúde da Família: pela integralidade da atenção e reorientação do modelo assistencial. Interface Comun Saúde Educ 2005; 9:39-52.

30. Gazzinelli MF, Gazzinelli A, Reis DC, Penna CMM. Educação em saúde: conhecimentos, representações sociais e experiências da doença. Cad Saúde Pública 2005; 21:200-6.

31. Mann J, Tarantola DJM. AIDS in the World II: global dimensions, social roots, and responses. New York: Oxford University Press; 1996.

32. Ayres J, França I, Calazans G, Salletti H. Vulnerabilidade e prevenção em tempos de AIDS. In: Barbosa R, Parker R, organizadores. Sexualidade pelo avesso: direitos, identidades e poder. Rio de Janeiro: Editora Relume-Dumará; 1999. p. 50-71.

33. Sabroza PC. Brasil deve aprender com epidemia de dengue no Rio. http://www.ensp.fi ocruz.br/portal-ensp/informe/materia/index. php? matid=9624\&origem $=4$ (acessado em 06/ Ago/2009).

Recebido em 04/Mar/2009

Versão final reapresentada em 19/Ago/2009

Aprovado em 09/Set/2009 